\title{
MedienPädagogik
}

Zeitschrift für Theorie und Praxis der Medienbildung

\section{Subjektorientierte Softwareentwicklung als medienpädagogische Herausforderung}

\author{
Interdisziplinäre Zugänge in den Digital Humanities
}

Bianca Burgfeld-Meise, Dorothee M. Meister und Björn Senft

\section{Zusammenfassung}

Die Medienpädagogik öffnet sich zunehmend interdisziplinären Zugängen und wird von unterschiedlichen Bereichen wie den Digital Humanities angefragt, ihre Expertise einzubringen. Dabei kennzeichnet die Digital Humanities eine intensive interdisziplinäre Zusammenarbeit und innovative wissenschaftliche Fragstellungen. Die Forschungssoftware, mit der diese innovativen Fragestellungen bearbeiten werden sollen, muss entsprechend flexibel sein, um sich mit den evolvierenden Aufgaben zu entwickeln. Dies birgt für die Softwareentwicklung in diesem Bereich vielfältige Unwägbarkeiten. Komplexitätssteigernd wirkt, dass hier nicht einfache Software für den Alltag erarbeitet werden muss. Vielmehr gilt es, digitalen Umgebungen für sich stetig weiterentwickelnde Forschungen gerecht zu werden und je nach Projektkontext unterschiedlichen Anforderungen zu genügen. Diese Anforderungen sind für Nicht-Softwareentwickler häufig nicht leicht zu verbalisieren. In diesem Beitrag stehen die interdisziplinäre Bearbeitung und Bündelung unterschiedlicher Expertisen im Vordergrund, um subjektorientierte Lösungen für solch komplexe Fragestellungen zu entwickeln. Der Beitrag diskutiert diese Themen beispielhaft anhand der interdisziplinären Projektarbeit der Medienforschung, der Medienpädagogik und der Informatik zur Softwareunterstützung digitaler Musikeditionen und somit der Verarbeitung von textuellen und nichttextuellen Daten. Hierbei steht die Methode zur Erforschung impliziten Wissens und deren Transfer für die Softwareentwicklung im Mittelpunkt der Diskussion. Daraus werden abschliessend medienpädagogische Empfehlungen für die Softwareentwicklung und für die Bildungsbedarfe in diesen Bereichen formuliert.

Subject-oriented software development as a challenge for media education

\begin{abstract}
Complex questions are currently discussed in the field of Digital Humanities. This does not only concern the cultural side of humanities but also the digital representation of cultural artefacts their processing, analysis and long-term availability. To handle all these facets, interdisciplinary collaboration is required. Software development in digital humanities is
\end{abstract}


one aspect within this discipline. Highly specialized software is developed for scientific research. This introduces an uncertain element when designing the software: the user group is characterised by expertise and their knowledge is therefore often implicit. Research with digital data in a digital environment being different from traditional research and its continual advancement increase the complexity even further. These issues have to be recognized by all participating research disciplines with DH context in order to make research contributions. The article discusses these topics using the interdisciplinary project work of media studies and computer science for software support of digital music editing and thus handling of textual and non-textual data. The method of researching the perspective and needs of the subjects and converting them to requirements are the focus of this discussion.

\section{Einleitung}

Die Digital Humanities (DH) befassen sich im Wesentlichen damit, innovative geisteswissenschaftliche Fragestellungen mittels informatischer Prozesse zu bearbeiten. Ein Beispiel dafür ist die Herausgabe einer Musik-Edition (etwa Beethovens Werke). Mit Unterstützung entsprechender Software kann die Arbeit des Editierens besser bewältigt werden; Verarbeitung und Verbreitung der Ergebnisse können einem grösseren Publikum zur Verfügung gestellt werden. Dabei handelt es sich noch um eine sehr junge Disziplin, die auf Interdisziplinarität angewiesen ist. Die DH sind ein Handlungsfeld, das in interdisziplinären Teams arbeitet und in dem neuerdings auch medienpädagogische Expertise gefragt ist. Diese kann sich auf unterschiedliche Bereiche konzentrieren. Diese erstrecken sich vom Anspruch, die komplexen Problematiken bei der Entwicklung technischer Systeme oder von Software-Entwicklungen zielgruppenorientiert mitzugestalten über den Anspruch, im digitalen Kontext (Selbst-)Bildungsprozesse besser zu verstehen bis hin zum Bemühen, angepasste und zielgruppenadäquate Bildungsprozesse für diesen Bereich zu konzipieren. Anhand eines Beispiels aus den Digital Humanities wird ein solches interdisziplinäres Vorgehen detailliert beschrieben. Welche Rolle medienpädagogischen Ansätzen in diesem interdisziplinären Kontext zukommen, soll im Folgenden näher erläutert werden. Dabei erscheint es unabdingbar, zunächst die Zugänge aller Disziplinen zum Thema nachvollziehen zu können.

Bei der Softwareentwicklung in Unternehmen sind nach wie vor unvollständige, verborgene und sich ändernde Anforderungen die am häufigsten genannten Probleme (Méndez Fernández und Wagner 2014). Innerhalb der Digital Humanities (DH) sind die Unwägbarkeiten noch herausfordernder, da diese immanent im Gegenstand verankert sind. Das resultiert zum einen aus dem innovativen Charakter der zu entwickelnden Lösungen, wie es Burdick et al. (2012) charakterisieren: 
"Digital Humanities work embraces iterative, in which experiments are run over time and become object to constant revision. Critical design discourse is moving away from a strict problem-solving approach that seeks to find a final answer: Each new design opens up new problems and - productively - creates new questions.» (Burdick et al. 2012, 22)

Durch die Tatsache, dass Forscherinnen und Forscher auf ihrem Gebiet durch Software unterstützt werden sollen, ist zum anderen eine enge Verzahnung von Technologien und geisteswissenschaftlichen Forschungsmethoden und Inhalten erforderlich. Hierfür ist sowohl Expertenwissen aufseiten der Forschung in den Geisteswissenschaften als auch auf der technologischen Seite notwendig. Allerdings bedeutet Expertenwissen, dass es sich nicht nur um explizites Wissen, sondern vielfach um implizites Wissen handelt, welches nicht ohne Weiteres zugänglich ist. ${ }^{1}$ Insofern sind Strategien notwendig, um insbesondere das implizite Wissen vor allem im Sinne von habitualisierten Arbeitsweisen, Orientierungen und Routinen offenzulegen (Gervasi et al. 2013), wie dies etwa auch in medienpädagogischen Kontexten häufiger realisiert wird (etwa Aßmann 2013).

Software Engineering und Usability Engineering haben bereits zahlreiche Methoden zur Nutzerinnen- und Nutzerforschung (u.a. mit Rekurs auf die Qualitative und Quantitative Sozialforschung) adaptiert. Das Interview (Holtzblatt, Wendell, und Wood 2004) wird für Gespräche mit Nutzerinnen und Nutzern eingesetzt, die Beobachtung (Zühlke 2012) um sie bei ihrer Tätigkeit zu begleiten und daraus Erkenntnisse zu gewinnen. Bisweilen kommt auch eine Kombination aus beiden Methoden zur Anwendung: das kontextuelle Interview (Aßmann 2013, 50-59), bei welchem die Nutzerinnen und Nutzer bei der Lösung bestimmter Aufgaben beobachtet und befragt werden. Diese Methoden bereichern die Entwicklung von Software, da die Anforderungen an den Befragten orientiert werden. Jedoch sind die genannten Disziplinen immer am Ergebnis orientiert. Das heisst Nutzerinnen und Nutzer werden befragt, um bisherige Software zu optimieren, vorhandene Anforderungen zu spezifizieren und zu benennen.

Diese Techniken sind im Bereich der Digital Humanities nur begrenzt geeignet, da Software neu konzipiert wird oder ungewiss ist, was die Software konkret leisten muss. Zudem muss bedacht werden, dass die Nutzerinnen und Nutzer solcher Software nicht die Optimierung der Software im Blick haben, sondern die Optimierung und Unterstützung ihrer Tätigkeiten und Routinen. Aus dem Designbereich gibt es den Ansatz des Design Thinkings, um den Menschen in den Vordergrund zu rücken und

1 Der Dualismus zwischen explizitem und implizitem Wissen stellt eine Komplexitätsreduktion tatsächlich vorhandener Wissensformen bis zu verschiedenen Varianten des Nicht-Wissens (vgl. etwa Riethmüller 2012). dar. Für die Erforschung von Nutzerbedürfnissen, deren Arbeitsroutinen und wissenschaftliche Praktiken erweist sich dieser Dualismus insbesondere in der Kollaboration mit der Informatik als forschungspragmatisch sinnvoll. 
aus Bedürfnissen Lösungen zu generieren (Kelley 2001). Hier wird ein experimentelles, iteratives Vorgehen vorgeschlagen. Diese ersten guten Ansätze sind jedoch noch nicht systematisch ausgereift. In dieser Hinsicht ist der Rekurs auf die empirische Sozialforschung sehr lohnend, da sowohl die quantitative als auch die qualitative Sozialforschung systematische Methodologien, Erhebungs- und Auswertungsmethoden bereithält, um regelgeleitet die Perspektiven von Individuen zu erforschen. Zudem gilt es zu bedenken, dass Methoden des Software Engineerings und des Usability Engineerings auf die Erstellung eines Produkts ausgerichtet sind. Und dies unter der Prämisse, dass die Nutzerinnen und Nutzer klar formulieren können wie ein Produkt aussehen soll. Allerdings führen Frohlich und Sarvas (2011) an, dass Technologie sowohl durch kulturelle Einflüsse auf die Entwickler als auch durch die potenziellen Nutzerinnen und Nutzer und deren kreativen Ansätze der Handhabung geformt wird. Die Identifizierung und Interpretation solcher sozialer Konstrukte wie auch die Offenlegung impliziten Wissens sind Prozesse, die eher durch sozialwissenschaftliche Verfahren erforscht werden können, die auch in der Medienpädagogik zur Anwendung kommen (Knaus 2017). Diese haben aber in den meisten Fällen keinen Bezug zu Fachwissen der Informatik. Somit sind vielfältige diskursive Prozesse notwendig, um in dieser Situation aus den Bedürfnissen der Nutzerinnen und Nutzer Anforderungen zu generieren, was insbesondere die qualitative Sozial- und Medienforschung leisten kann. Die Expertise des Usability Engineerings und des Software Engineerings sind im Anschluss notwendig, um diese empirischen Erkenntnisse in Anforderungen zu überführen.

Das vom BMBF geförderte Digital Humanities Projekt «Zentrum Musik - Edition - Medien» (ZenMEM) stellt sich diesen Herausforderungen und entwickelt hierfür Lösungen in einem interdisziplinären Kontext. Im Vordergrund steht u.a. die Unterstützung von Musikwissenschaftlern bei ihrer Arbeit als Editoren mit der Forschungssoftware Edirom. ${ }^{2}$ Diese erlaubt es, Faksimiles, Digitalisate und digitale Daten von Notentexten oder anderen Quellen zu speichern, zu organisieren, zu kollationieren, zu annotieren und zu analysieren. Musikwissenschaft, Software Engineering, Usability Engineering, quantitative und qualitative empirische Medienforschung forschen hier gemeinsam an der Erarbeitung neuer Modelle für die Editionssoftware Edirom. An dieser wird im Folgenden das Vorgehen und die damit verbundenen Herausforderungen exemplarisch verdeutlicht. Die Problemstellung ist darüber hinaus relevant, da grundsätzliche Fragestellungen von Unsicherheiten in der Softwareentwicklung bearbeitet werden.

Zudem wird dargelegt, wie die Bereiche durch Synthese der fachdisziplinären Zugänge, Methoden und Ergebnisse diese Problemstellungen im Rahmen der frühen Anforderungsanalyse konzipiert und umgesetzt werden. So verschafft sich die quantitative Medienforschung einen Überblick über den gesamten potenziellen Kreis der

2 Siehe hierzu auch http://www.edirom.de [11.05.2020]. 
Nutzerinnen und Nutzer. Die qualitative Medienforschung analysiert das implizite Wissen individueller Nutzerinnen und Nutzer. Das Software Engineering erarbeitet durch die Erhebung von stabilen Anforderungen neue Modelle für die Softwareentwicklung. Das Usability Engineering optimiert Oberflächengestaltung und darunterliegende Softwarearchitektur und die Medienpädagogik leitet aus den Ergebnissen Gestaltungsempfehlungen für die Software sowie Empfehlungen für Bildungsmassnahmen ab. Um diese Perspektiven systematisch zu verbinden, sind Kollaboration und Synthese zwischen den Disziplinen notwendig.

\section{Forschungsansatz und -methode}

Im interdisziplinären Forschungsprozess ist es notwendig, die unterschiedlichen Perspektiven und Verfahren methodologisch zusammenzuführen und somit systematisch miteinander zu verbinden. Aufgrund ihrer Offenheit wurde die Grounded Theory Methodologie (GTM) gewählt, die auch in der Medienpädagogik inzwischen häufig verwendet wird (Aßmann 2013; Kamin 2017), um nachhaltige Anforderungen zu erarbeiten. Die GTM setzt ursprünglich die Reziprozität von Theorie und Empirie voraus (Strauss und Corbin 1996). Die Forschungselemente Theorie und Datenerhebung sowie deren Analyse stehen in ständiger Wechselbeziehung, beeinflussen sich gegenseitig und tragen durch eine sinnvolle und nachvollziehbare Kombination zur Entwicklung einer empirisch fundierten Theorie bei. Damit stellt sie ein iteratives Verfahren dar, das insbesondere zur Bearbeitung dynamischer Forschungskontexte wie im Fall der Digital Humanities geeignet ist. Die Grounded Theory Methodologie bietet viele Freiheiten, den Forschungsprozess zu gestalten. Je nach Forschungsphänomen und individueller Forschungsperspektive können so sehr heterogene Konzeptionen und Variationen entworfen werden (Mey und Mruck 2011). Da methodologisch zunächst die sinnvolle Kombination von Theorie und Empirie im Vordergrund steht, muss der Ansatz nicht auf eine Disziplin beschränkt sein. Vielmehr bietet sich hier die Möglichkeit, verschiedene theoretische sowie empirische Perspektiven aus den beteiligten Fachdisziplinen auf die Problemstellung anzuwenden und somit die Anforderungen sukzessiv immer besser zu beschreiben. Dementsprechend ist es nicht, wie bei herkömmlichen GTM-Konzeptionen das Ziel, eine Theorie zu entwickeln, sondern nachhaltige Anforderungen an die Softwareentwicklung zu konkretisieren.

Um die Bedeutung von Medien, in diesem Fall die Nutzung der Forschungssoftware Edirom, erschliessen zu können, ist es notwendig, die vielschichtigen Auseinandersetzungsprozesse der Nutzerinnen und Nutzer mit der Software zu erforschen. Damit verbunden ist die sogenannte radikale Kontextualisierung in den Cultural Studies, bei der davon ausgegangen wird, dass «Objekt und Subjekt, Medientechnologie und Kontext» (Winter 2010) sich stetig beeinflussen und miteinander verwoben sind. 
Aufgrund der Komplexität der Perspektiven kann im Projekt nicht die eine Methode für alle Bereiche angewandt werden. Vielmehr ist die sinnvolle Synthese kumulativer Methoden notwendig, um dieser Komplexität Rechnung zu tragen. Nachfolgend werden zuerst die fachdisziplinären Methoden mit ihren spezifischen Sichtweisen sowie ihrer Relevanz für die Anforderungsanalyse verdeutlicht.

\section{Interdisziplinäres Vorgehen}

Durch die enge Verknüpfung der verschiedenen Software-Schichten in der bisherigen Editionssoftware Edirom liessen sich in einer Analyse im Rahmen einer Reverse Engineering Phase nur die Dokumentation der angebotenen Funktionalitäten und erste strukturelle Schwächen des verwendeten Datenmodells identifizieren und dokumentieren. Daher wurde trotz bestehender Software kein Reverse Engineering Ansatz, sondern ein Ansatz zur Neu-Entwicklung des Edirom-Editors verfolgt. Aufgrund der einleitend angeführten Herausforderungen haben sich keine geeigneten Methoden gefunden, um eine systematische Software-Entwicklung mit relativ sicheren Anforderungen zu beginnen. Bestehende Ansätze wie die agile Software-Entwicklung oder auch Lean Software Development stellen zwar einen Ansatz dar, um möglichst frühzeitig Rückmeldungen von Nutzerinnen und Nutzern einzubeziehen. Allerdings liegt ihr Fokus auf lauffähiger Software und der Reduzierung von «Müll» bzw. Tätigkeiten (Meyer 2014). Im Fokus steht nicht die frühzeitige systematische Identifizierung impliziter Anforderungen, sondern die nachträgliche Evaluation, ob ein Inkrement vom Kunden akzeptiert wird. Für den Bereich der Anforderungen hat sich die Disziplin des Requirements Engineering etabliert, das nach Hickey und Davis (2004) durch die Aktivitäten Erhebung, Analyse, Triage, Spezifizierung und Verifikation beschrieben werden kann. Vor allem für die Erhebung steht auch diese Disziplin vor Herausforderungen, da bestehende Ansätze vor allem auf die Exploration des Raumes bekannter Anforderungen abzielen und nicht auf die Erhebung neuer Anforderungen (Sutcliffe und Sawyer 2013; Maiden et al. 2010). Als Lösung hierfür wird die Einführung von kreativen Methoden genannt.

Die Erkenntnisse aus dem Anforderungsmodell boten den Mitarbeiterinnen der empirischen Medienforschung einen Überblick über die wichtigsten Funktionalitäten des Programms und somit erste Anhaltspunkte für Fragedimensionen für den quantitativen Fragebogen und den qualitativen Leitfaden. Eine weitere wichtige Rolle bei der Erstellung des Fragebogens spielte auch die musikwissenschaftliche Hospitation bei einem Experten, der die verschiedenen Herangehensweisen der wissenschaftlichen Arbeit von Musikeditoren insbesondere anhand der Forschungssoftware Edirom erläuterte. Ergänzend dazu wurde bei der Konstruktion der Fragen auf Fachliteratur (Feder 1987) zurückgegriffen. Fragen bezüglich der Software- und Technikaffinität sind angelehnt an die Items für Konstrukte des Technology Acceptance Model 
III (Venkatesh und Bala 2008). Ergänzt wurde der Fragebogen um einzelne Fragen, die konkrete Features in der Edirom Forschungssoftware bei den Befragten abfragen.

In der quantitativen steht im Gegensatz zur qualitativen Medienforschung in einer Befragung nicht das Individuum im Zentrum des Interesses, sondern eine Population (Grundgesamtheit), über welche der oder die Forschende Aussagen treffen will (Müller-Benedict 2007). Für die vorliegende Befragung wurde die Online-Befragung in Form eines Surveys (Querschnittsstudie) durch eine gezielte Ansprache per Mail als geeignete Methode bestimmt, was hauptsächlich darin begründet ist, dass es sich bei der Zielgruppe um eine fachlich spezielle, geografisch weit verteilte Personengruppe handelt. Dafür wurde die Einladung zur Befragung über den E-Mail-Verteiler der «Fachgruppe Freie Forschungsinstitute» (FFFI) versandt. Zur FFFI gehören derzeit 50 Institute mit rund 140 wissenschaftlichen Mitarbeiterinnen und Mitarbeitern. Davon arbeitet etwa die Hälfte an Musikeditionen. Bestandteil des Fragebogens waren insgesamt 41 Fragen zu vier grossen Themenkomplexen. Im ersten Komplex standen zunächst die wissenschaftliche Arbeit und editorische Tätigkeit im Fokus. Im zweiten Themenkomplex ging es um die Forschungssoftware Edirom sowie die Art und Weise, wie sie im Rahmen der wissenschaftlichen Arbeit eingesetzt wird, beziehungsweise warum sie vielleicht auch nicht genutzt wird. Der dritte Teil des Fragebogens erhob das Engagement der Befragten in der externen Wissenschaftskommunikation und ihre Meinung zu dieser aus der Perspektive ihres Fachgebiets. Zum Abschluss wurden die soziodemografischen Daten erhoben sowie Angaben zu ihrem musikalischen Hintergrund und ihrer Technikaffinität.

Qualitative Medienforschung birgt den Vorteil, in einem unbekannten Forschungsfeld einsetzbar zu sein. Parallel zur quantitativen Forschung wurde im Projekt ein hypothesengenerierendes und exploratives Verfahren angewandt. Erhebt also die quantitative Forschung Daten zu vielen Nutzerinnen und Nutzer, um Überblicke zu generieren und Themenfelder zu identifizieren, ist die qualitative Forschung dazu in der Lage, in die Tiefe zu gehen und bspw. implizites Wissen, unbewusste Routinen oder auch habitualisierte Arbeitsweisen zu eruieren. Dabei ermöglicht qualitative Medienforschung, nicht nur Stichworte, sondern Kontextinformationen und bislang unbekannte Dimensionen aus den Daten herauszuarbeiten (Flick 2002). Um die Sicht der Editorinnen und Editoren zu erschliessen, hätten sich unterschiedliche Methoden angeboten wie Beobachtung, unterschiedliche Arten von Interviews, Einzel- bzw. Gruppeninterviews etc. (vgl. etwa Flick, Kardorff, und Steinke 2000). Die Auswahl der Erhebungsmethode begründet sich nach dem Forschungsphänomen. Die Erforschung impliziten Wissens, von Arbeitsroutinen, Expertisen und Gewissheiten lässt sich kaum über einzelne direkte Fragen realisieren. Um solche Phänomene aufzuspüren ist zunächst der Gesamtkontext wichtig. Das implizite Wissen als Teil des gesamten Wissenssystems wie Polyani (1985) es entwarf, beschäftigt auch Neuweg (1999). Er verdeutlicht die Relevanz des Kontextes mit einem Verweis auf Wittgenstein: 
«Wenn wir anfangen etwas zu glauben, dann nicht einen einzelnen Satz, sondern ein ganzes System von Sätzen. (Das Licht geht nach und nach über das Ganze auf.)» (Wittgenstein 1984)

In diesem Sinne nutzt die qualitative Medienforschung verschiedene Formen von Befragungstechniken, um unterschiedliche Arten von Narrationen zu erhalten, die im Anschluss verschriftlicht und analysiert werden können. Im Auswertungsprozess wird dann über Interpretationen der Gesamtkontext erarbeitet und erschlossen. Dies geschieht, indem einzelne Wissensbestände mit anderen Aussagen verbunden werden, die im Gesamtkontext Einblicke in das Zusammenwirken expliziter und impliziter Wissensbestände erlauben. Als Erhebungsform für diese qualitative Studie wurde das narrative Leitfadeninterview mit problemzentrierten Anteilen gewählt, das zwar einem Leitfaden folgt, in der Interviewsituation allerdings grösstmögliche Spielräume hinsichtlich der Frageformulierungen, Nachfragestrategien und der Reihenfolge der Fragen zulässt (Keuneke 2005) und der Narration der Befragten viel Raum gibt. Der Leitfaden fokussierte besonders Regelstrukturen der Handlungs- und Nutzungsweisen, indem die Befragten nach bestimmten Nutzungssituationen und den damit verbundenen Bedeutungen und Relevanzen befragt wurden. Diese Nutzungssituationen und Bedeutungen wurden allen interviewten Personen gleichermassen vorgegeben. Die von den Befragten formulierten Antworten konnten anschliessend aufeinander bezogen werden. Im Sinne des Theoretical Sampling (Przyborski und Wohlrab-Sahr 2009) wurden Expertinnen und Experten befragt, die mit Edirom arbeiten. Entscheidend war, dass sowohl Nutzerinnen als auch Nutzer befragt wurden. Insgesamt wurden acht Interviews mit sechs Editorinnen und zwei Editoren geführt. Diese dauerten zwischen 90 und 180 Minuten. Die erhobenen qualitativen Daten wurden durch eine Variante des Kodierens nach Strauss und Corbin ausgewertet, wie es Przyborski und Wohlrab-Sahr (2009) vorschlagen. Die Auswertung wurde durch Textverarbeitungsprogramme unterstützt, um die sich herausbildenden Phänomene einer exemplarischen, interdisziplinären Sichtung unterziehen zu können. In der Synthese der exemplarischen Auswertung konnte das selektive Codieren vorangetrieben werden, woraus eine Phänomen- und Kategorienliste resultierte. Diese liefert einerseits konkrete Hinweise für Optimierungen der Forschungssoftware, aber auch Kontextinformationen zu den Arbeitsbedingungen, Routinen und Erfahrungen der Editorinnen und Editoren. Darüber hinaus liefern die Interviews sehr gute Einblicke in die Änderungsprozesse der Wissensarbeit, des Wissensmanagements wie auch des erarbeiteten Wissens überhaupt. All diese Erkenntnisse tragen dazu bei, die Nutzerinnen und Nutzer besser einschätzen und unterstützen sowie zudem die Editionen weiterentwickeln zu können. 
Sowohl im Requirements Engineering (Pohl 2007) als auch im Usability Engineering (DIN 9241) wird eine Beschreibung der Nutzerinnen und Nutzer als essenziell angesehen. Diese muss in verschiedene Modelle für die verschiedenen Nutzungstypen differenziert werden (Cooper, Reimann, und Cronin 2007). Modelltypen, die hierfür verwendet werden können, sind beispielsweise Benutzerinnen- und Benutzerrollen oder Persona. Benutzerinnen- und Benutzerrollen nach Constantine und Lockwood (1999) sind in ihrer einfachsten Form Listen von Eigenschaften wie Bedürfnisse, Interessen, Erwartungen, Verhalten und Verantwortlichkeiten. Sie sind nicht als Abstraktion von realen Personen gedacht. Daher werden Personae (Cooper, Reimann, und Cronin 2007) für die Modellierung von Nutzerinnen und Nutzern verwendet. Diese basieren auf Beobachtungen und stellen keine Stereotype, sondern Archetypen dar. Unterschieden werden muss zwischen den eigentlichen und vorläufigen Personae, da bei der letzteren Variante die Datenbasis für eine fundierte Erstellung einer Persona nicht ausreichend ist. Um eben diese Typenbildung für Personae zu nutzen, ergänzen sich Usability Engineering und empirische Sozialforschung hervorragend. So sind kategorisierende Analyseverfahren in der quantitativen und qualitativen Sozialforschung ebenso üblich wie im Usability Engineering. Aus der quantitativen Analyse sind zunächst keine spezifischen Typen von Nutzerinnen und Nutzern ableitbar. In der qualitativen Forschung stellt sich das anders dar. Mit den Erkenntnissen aus den Interviews (Bohnsack 2010) und den Tests aus dem Usability Engineering wurde interdisziplinär an der konkreten und detaillierten Beschreibung von Personae gearbeitet. Dies ermöglicht noch einmal eine eingehendere Auseinandersetzung mit dem impliziten Wissen der Nutzerinnen und Nutzer, ihrer Handlungsroutinen und Orientierungen. Daraus ergibt sich eine Wissensbasis und ein Wertverständnis, das die Grundlage sowohl für eine weitere iterative Betrachtung und Validierung der Ergebnisse als auch für die Entwicklung von potenziellen Lösungen mithilfe des Design Thinkings darstellt. Startpunkt des Design Thinkings ist die sogenannte Design Challenge, welche den Rahmen festlegt, in dem operiert wird (vgl. IDEO LLC. 2013; Schallmo und Lang 2017). Sollte dieser Rahmen zu breit, zu vage, zu eingeschränkt oder auf Basis eines falschen Wertverständnisses formuliert sein, würden die hieraus resultierenden Softwareprodukte nicht zuverlässig genug an den Subjekten und ihren Werten ausgerichtet sein. Das Design Thinking stellt allerdings keine Methoden bereit, um solch eine Design Challenge zu identifizieren, sondern nur, um diese post factum auf ihre Validität hin zu überprüfen. Hier zeigt sich die Zusammenarbeit mit der empirischen Medienforschung als besonders fruchtbar, da die Informatik hiermit in der Lage versetzt wird, ein valides Wertverständnis initial aufzubauen und dieses in eine Design Challenge zu übersetzen, um so mithilfe des divergierenden und konvergierenden Denkens sowie des iterativen Explorierens des Problem- und Lösungsraums des Design Thinkings Lösungen und Anforderungen zu entwickeln, die sich an den Werten der Subjekte ausrichten. Zudem lässt sich so der GTM-Ansatz in die 
Produktentwicklung weiterziehen und die aus der initialen empirischen Vorgehensweise gewonnenen Theorien und Erkenntnisse lassen sich tiefgehender durch die Umsetzung in Produkten und deren Praxistest tiefergehender validieren. In Anlehnung an Design Based Research nach Reinmann (2005) lässt sich das beschriebene Vorgehen ebenfalls als einen Lernprozess fassen, in dem nützliche Innovationen für den musikwissenschaftlichen Kontext erschaffen werden. Im Fokus steht dabei ein stetiges Zusammenspiel von «believe mode» und «design mode». Ohne Vorwissen lassen sich keine nützlichen Innovationen designen und ohne Design und Verwendung in der Praxis lassen sich Theorien nur auf einer diskursiven Ebene bewerten. Erst durch eine Verwendung in einem praktischen Kontext wird sichergestellt, dass alle Dimensionen zutage treten können. Vorher handelt es sich immer nur um Gedankenexperimente, die geeignet sind, aus Unkenntnis oder Fehlerhaftigkeit entstandene Elemente unter Laborbedingungen zu eliminieren, obwohl sie einen wichtigen Einfluss auf die Validität der Theorie haben.

\section{Ergebnisse}

Im Folgenden werden nun einige exemplarische Ergebnisse aus dem zuvor dargestellten interdisziplinären Vorgehen skizziert. Aus der quantitativen Forschung wurden zunächst grundlegende Erkenntnisse über die Editorinnen und Editoren erarbeitet. Diese beschreiben sich selbst als sehr technikaffin («Es fällt mir leicht, den Umgang mit neuen technischen Geräten zu erlernen» - Zustimmung von 82,5\%) und softwareaffin («Ich habe keine Angst, neue Software zu nutzen»- Zustimmung von $82,4 \%)$. Sie arbeiten mehrheitlich in dezentral strukturierten Projekten, was auf spezielle Bedürfnisse in Bezug auf die Kommunikation und Kollaboration innerhalb des Projekts hindeutet.

In den qualitativen Interviews werden die Konsequenzen des dezentralen und kollaborativen Arbeitens noch deutlicher: Dadurch, dass bestimmte Programme zu teuer sind oder aber der dezentrale Datenaustausch mit den Servern nicht reibungslos läuft, wird im Homeoffice auch mit Papier und Bleistift oder Word gearbeitet und das Ergebnis so bald wie möglich in Edirom eingepflegt. Darüber hinaus nutzen Editorinnen und Editoren viele verschiedene Softwareprodukte für ihre editorische Tätigkeit, wogegen die Mitarbeiter in Projekten analoger Editionen kaum softwaregestützt arbeiten. Diese Erkenntnisse lassen sich qualitativ ergänzen. Editorinnen und Editoren recherchieren im Rahmen ihrer analogen Tätigkeit vor Edirom zunächst Quellen, analysieren diese und wählen dann Haupt- und Nebenquellen aus, um die weitere Editionsarbeit zu gestalten. Im Anschluss daran wurden diese Quellen stetig miteinander verglichen. Dazu musste sehr viel Quellenmaterial physisch verwaltet werden, um die einzelnen Änderungen in der jeweiligen Quelle mit anderen vergleichen und analysieren zu können. Die Arbeit an den digitalen Editionen ist indes ein 
Konglomerat aus analogen und digitalen Techniken. Zuerst werden die Quellen ebenso recherchiert, analysiert, ausgewertet und ausgewählt. Die ausgewählten Quellen werden von den Hilfskräften im Anschluss digitalisiert, vertaktet, ${ }^{3}$ anschliessend werden die Konkordanzen ${ }^{4}$ festgelegt. Es findet also eine Arbeitsteilung statt, da die Vertaktung delegiert wird. Dieser Prozess konnte somit nicht durch die Interviews eruiert werden und erforderte entsprechend weitere empirische Verfahren. Da die Vertaktung ein sehr standardisierter Prozess ist, konnte dieser mit entsprechenden kontextuellen Interviews erforscht und für das Usability Engineering ausgewertet werden. Innerhalb der Erhebung wurden die Hilfskräfte bei ihrer Vertaktungsarbeit beobachtet und befragt. Die so gewonnenen Daten sind in die Entwicklung eines Vertaktungswerkzeugs eingeflossen, welches die halbautomatische Vertaktung erlaubt und somit eine Unterstützung für die Arbeit der Hilfskräfte darstellt und den Zeitaufwand erheblich verringert.

Die Interviews verweisen derweil auf die hohen Vorteile und Freiheitsgrade der digitalen Editionen, da den Rezipientinnen und Rezipienten nun erstmals das gesamte Quellenmaterial zur Verfügung gestellt werden kann. Diese können nun editorische Entscheidungen transparent nachvollziehen und eine eigene Meinung dazu entwickeln. Damit einhergehend ist aber auch ein zunehmendes Mass an Komplexitätssteigerung und wachsenden Aufgaben zu verzeichnen. Bei Printeditionen steht die editorische Tätigkeit im Fokus und ist durchaus anspruchsvoll. Der Wechsel zur digitalen Edition bedeutet für die Editorinnen und Editoren einen weiteren Komplexitätsschub: Nicht nur die musikwissenschaftliche Expertise ist gefragt, sondern auch Kenntnisse verschiedenster Auszeichnungssprachen, wie XML, TEI und MEI. Aufgrund der Arbeitsteilung muss darüber hinaus den Hilfskräften Wissen für die Vertaktung vermittelt und müssen diese angeleitet und kontrolliert werden. Zudem nutzen die Editorinnen und Editoren, wie bereits im quantitativen Teil herausgearbeitet, notwendigerweise zusätzliche Programme. Um nur einige zu nennen: Sibelius, Score, Finale, QuarkX, InDesign, OxygenXML, LilyPond, Word, Filemaker, oder aber Verovio. Hier gilt es zu überlegen, ob zur Unterstützung systematische Schnittstellen oder aber Konverter entwickelt werden, um die Editorinnen und Editoren zu entlasten. Wie die aufgeführten Notensatzprogramme verdeutlichen, sind Editorinnen und Editoren auch mit Aufgaben beschäftigt, die vorher von Verlagen erledigt wurden. Durch

3 Unter vertakten versteht man in Editionskontexten das Festlegen der Taktgrenzen. Bei der Digitalisierung werden auf dem digitalisierten, vormals analogen Material digital die Taktgrenzen vermerkt und gezählt. Dieser Vorgang wird für alle Schriften (Bearbeitungen der Komponisten, Kopisten, Setzer des Verlags etc.) und alle Stimmen des Werkes vorgenommen. Dies ist insbesondere notwendig, um später Konkordanzen herzustellen.

4 Konkordanzen werden gesetzt, indem unterschiedliche Kompositionsschriften miteinander verknüpft werden. Bspw. der Takt 1 in der Schrift des Komponisten (Autograph) mit dem Takt 2 des Setzers aus dem Verlag. Wenn diese miteinander verbunden werden, ist Takt 1 im Autograph der gleiche Abschnitt in der Komposition wie der Takt 2 des Verlags. Diese kann man sich dann gemeinsam anzeigen lassen, vergleichen, analysieren und dergleichen mehr. 
diese Tätigkeit kann auch das Rechtemanagement von Originalquellen zu einem weiteren Aufgabengebiet werden. Diese Ergebnisse zeigen sich in der Auswertung des offenen und selektiven Kodierens. Vertiefte Einsichten ergeben sich jedoch vor allem durch eine Sonderauswertung im Hinblick auf zentrale Orientierungs- und Bildungsmuster, die im Folgenden dargestellt wird:

«Also einmal die Arbeit als Editor verändert sich, indem du halt immer mehr Dinge jenseits der klassischen Editionsarbeit abarbeiten musst, mit rechtlichen Geschichten, wie bilde ich das ab, wie zeige ich, wie ich das abgebildet haben möchte. [...] Also da kommt ja immer mehr Expertise, die man in verschiedenen Berufsgruppen vorher hatte, laufen im Prinzip bei den Editoren zusammen.» (Editorin A, Z. 935-951).

Editorin A gehört zu denjenigen, die schon sehr lange computergestützt arbeiten. Dabei kam sie durch Eigeninitiative im Studium (ab 2001) dazu, Noten mittels Computer zu setzen. Daraus ergab sich ein lukrativer Nebenjob, da viele digital gesetzte Noten haben wollten, die Umsetzung aber nicht selbst bewerkstelligen konnten. So hatte $A$ an ihrem Studienort eine Sonderstellung, weil sie diese Fähigkeiten hatte und dies auch wahrgenommen wurde. Insgesamt lässt sich dieser Bildungsbiografieabschnitt als intrinsisch motivierter Lernprozess mit einer sehr hohen Selbstwirksamkeitserfahrung erfassen, der dafür sorgte, dass sich A weiterhin mit den Themen auseinandersetzte. Diese positiven Aspekte lassen sich ebenso zu der Zeit beobachten, als sie ihr eigenes Editionsprojekt autark betreute. Durch die individuell mögliche Arbeitsweise sind für A Ideen, Innovationen und Weiterentwicklungen möglich. Die Unterstützung durch Experten vor Ort empfindet sie dabei als sehr bereichernd.

In den anschliessenden Projektkontexten (ab 2009) mit den ersten Berührungspunkten zur Forschungssoftware Edirom ergaben sich dann Herausforderungen für A. Hier musste sie sich in grossen Projektkontexten zurechtfinden, arbeitsteilig organisierte Aufgaben übernehmen und letztlich auch die digitale Editionsarbeit mit den Experten vor Ort teilen. Auf dieser Stufe lässt sich ein Komplexitätsschub feststellen, da nicht mehr nur Noten digital gesetzt werden, sondern die digitale Editionsarbeit voranschreitet. Hier ist auch anzumerken, dass es damals noch unklar war, wie sich digitale Editionsarbeit entwickeln würde. Zu diesem Zeitpunkt wurde noch diskutiert, ob nur das Ergebnis digital ediert wird oder in Gänze digitale Editionsarbeit geleistet werden soll.

Strukturelle Bildungswiderstände lassen sich in den verschiedenen Wissensständen in Projekten feststellen. Nicht alle Wissenschaftlerinnen und Wissenschaftler verfügen gleichermassen über musikalische sowie technische Expertise. Um aber digitale Editionsarbeit erfolgreich zu gestalten, ist beides notwendig. Dies führt zu unangenehmen Erfahrungen für Wissenschaftlerinnen und Wissenschaftler, wenn bislang geschätzte Expertise nicht mehr ausreicht und Defizite im Technikwissen 
für Probleme sorgen. Das ist für Editorinnen und Editoren, die in ihrem Bereich ein grosses Wissen aufweisen können, eine schwierige Situation. Versuch und Irrtum bestimmen dann die technische Umsetzung - eine Arbeitsweise, die sonst kaum angewandt werden muss und dementsprechend mit Unsicherheiten belegt ist. Da es kaum eine Dokumentation zu Edirom gab bzw. wenig schriftlich aufbereitetes Wissen zu digitalen Editionen, haben es Editorinnen und Editoren schwer, sich autark Wissen anzueignen, um diese Defizite auszugleichen. In Ihrer Bildungsbiografie ist aber genau dies der primäre Zugang. Darüber hinaus verändern sich digitale Editionen und die Arbeitsschritte zu deren Erstellung und Repräsentation sehr dynamisch und projektspezifisch. Hier sind kommunikative Unterstützungsstrukturen wünschenswert, die eine einfache Informationsgewinnung über aktuelle Projekte und digitale Techniken erlaubt. Zu den strukturellen Widerständen zählen vor allem unklare Arbeitsverteilungen in grossen Projekten bzw. die Arbeitsteiligkeit des Prozesses. Hier scheint es Defizite in der Projektführung und -begleitung zu geben.

Für die Konzeption von Forschungssoftware ergibt sich dadurch die Anforderung, Selbstwirksamkeitserfahrungen und Autonomie zu stärken: Editorinnen und Editoren bearbeiten ein sehr spezialisiertes Feld der musikwissenschaftlichen Forschung. Neben Ihrer fachlichen Expertise gewinnen zunehmend digitale Fähigkeiten an Bedeutung. Um diese Zielgruppe anzusprechen, ist neben einer möglichst intuitiven Gestaltung von Forschungssoftware auch der Zugang zu Informationen und Dokumentationen zur Forschungssoftware zu digitalen Techniken etc. sinnvoll, damit eventuell vorhandene Wissenslücken auch autark behandelt werden können. Zudem gestaltet sich Editionsarbeit als dynamisch, arbeitsteilig und vielschichtig. Die Repräsentation des Projektstandes mit seinen arbeitsteiligen Anteilen könnte den Editionsprozess wesentlich besser nachvollziehbar gestalten und somit eine Orientierungshilfe zur Projektsteuerung anbieten. Die Implementation eines kommunikativen Austauschs kann dabei helfen, Absprachen einfacher und objektspezifischer zu gestalten. Darüber hinaus wäre ein übergeordneter Austausch der Beteiligten an Editionsprojekten zu neuen Entwicklungen und Projekten wünschenswert.

Die Ergebnisse abstrahierend betrachtet, sind im neuen Medium neue Forschungsfragen entstanden und bilden sich täglich neu aus: Die Anfangs- und Endpunkte von Editionen sind immer noch auszuloten, welche Nachnutzbarkeit kann gewährleistet werden, wie kann die Praxis von dem Wissen profitieren und dieses einsehen, wo ist Wissen gesichert erschlossen? Zudem gibt es kaum verbindliche Standards im digitalen Editionsprozess - was nun bei steigender Editionszahl und entsprechender Annotationsmenge immer offensichtlicher und drängender wird. Angesichts dieser Ergebnislage und deren interdisziplinärer Sichtung gibt es viele Anhaltspunkte wie etwa Schnittstellen und Konverterlösungen, um die Arbeit der Editorinnen und Editoren konkret zu unterstützten. Die Kontextinformationen der veränderten Arbeitsstrukturen, Aufgaben und Wissensbestände verweisen zudem auf die 
grundsätzliche Relevanz der (möglichst einfachen) Softwareunterstützung, um diese Herausforderungen bearbeiten und repräsentieren zu können. Ein systematischer Aufbau informatischer Grundkenntnisse wird ebenso implizit evident, um die Potenziale der digitalen Repräsentations- und Verarbeitungsoptionen besser erschliessen zu können. Hierzu sind spezifische Ausbildungskonzepte, Schulungen, Austauschplattformen und Online-Lernangebote notwendig, um den Editorinnen und Editoren gute Orientierungsmöglichkeiten in ihrem komplexen Berufsfeld zu bieten.

\section{Fazit und Ausblick}

In der Synthese der disparaten Zugänge zeichnen sich die Herausforderungen solcher Kollaborationen wie bspw. die Erarbeitung eines konsensuellen Vokabulars und eines ausreichenden gemeinsamen Wissenstandes ab. Gleichzeitig erweitern sich jedoch die Perspektiven und führen zu innovativen Forschungsansätzen. So wurden die Ergebnisse der Software Engineering Analyse und der musikwissenschaftlichen Hospitationen in ein Aufgabenmodell der Medienpädagogik überführt und durch Musikwissenschaftler evaluiert. Diese Ergebnisse sind dann in die Konzeption der Fragebögen und Leitfäden eingeflossen. Durch die interdisziplinäre Sichtung des empirischen Materials ergaben sich gerade im Hinblick auf Personae in Verbindung mit Verfahren der rekonstruktiven Sozialforschung detailliertere Arten der Auswertung, die jeweils fachdisziplinär nicht möglich wären. Insgesamt, so die ersten Ergebnisse, führt dieser interdisziplinäre Zugang trotz hoher Aufwände auf allen Seiten zu neuen und spannenden Erkenntnissen und diese tragen dazu bei, die nachhaltigen Anforderungen für die Softwareentwicklung umzusetzen. Besonders hervorzuheben ist dabei, wie sich durch unterschiedliche Zugänge andere Perspektiven auf Software und Nutzende ergeben und somit im Verbund Forschungssoftware subjektorientiert gestaltet werden kann.

Die Medienpädagogik konnte aus dem Projektgeschehen wichtige Erkenntnisse generieren. Aufmerksam gemacht werden konnte darauf, dass sich das wissenschaftliche Arbeiten der Editorinnen und Editoren durch die Digitalisierung dramatisch verändert hat. Das Handlungsfeld selbst wird komplexer aufgrund sehr unterschiedlicher Ansprüche aus Musik, Informatik und Projektmanagement. Hier besteht die Gefahr einer Überforderung der Handlungspraxen, die nur durch eine Veränderung der Weiterbildung und der Ausbildung abgemildert werden kann.

Angeregt werden konnte darüber hinaus eine neue Konzeption der EdiromSummer-School, die eine stärkere Einbeziehung der Nutzerbedürfnisse in das Fortbildungskonzept sicherstellt. Angestrebt wird, umfangreiche Selbstlernmaterialien zu etablieren, damit die Kommunikations- und Wissensbedürfnisse dieser speziellen Nutzergruppe besser befriedigt werden können. Für die Medienpädagogik ergibt sich daraus die Erkenntnis, dass in zahlreichen wissenschaftlichen und praxisnahen 
Handlungsfeldern grosser Bedarf besteht, sich die speziellen digitalen Praxen genauer anzusehen und am besten gemeinsam mit Informatikern und Praktikern entsprechende Softwarelösungen zu finden sowie Bildungskonzepte zu entwickeln.

\section{Literatur}

Aßmann, Sandra. 2013. Medienhandeln zwischen formalen und informellen Kontexten: Doing Connectivity. Medienbildung und Gesellschaft. Wiesbaden: Springer Fachmedien Wiesbaden. https://doi.org/10.1007/978-3-658-01940-2.

Bohnsack, Ralf. 2010. Rekonstruktive Sozialforschung: Einführung in qualitative Methoden. Opladen u.a.: Barbara Budrich.

Burdick, Anne, Johanna Drucker, Peter Lunenfeld, Todd Presner, und Jeffrey Schnapp. 2012. DIGITAL HUMANITIES. Cambridge, Massachusetts: MIT Press.

Constantine, Larry L. und Luca A. D. Lockwood. 1999. Software for Use: A Practical Guide to the Models and Methods of Usage-Centered Design. Boston, San Francisco, New York et al: Addison-Wesley Professional.

Cooper, Alan, Robert Reimann, und David Cronin. 2007. About Face 3: The Essentials of Interaction Design. Indianapolis: Wiley Publishing. https://fall14se.files.wordpress.com/2017/04/ about_face_3_the_essentials_of_interaction_design.pdf.

Schallmo, Daniel R. A., und Klaus Lang. 2017. Design Thinking erfolgreich anwenden. Wiesbaden: Springer Gabler.

Feder, Georg. 1987. Musikphilologie: Eine Einführung in die musikalische Textkritik, Hermeneutik und Editionstechnik. Darmstadt: Wissenschaftliche Buchgesellschaft.

Méndez Fernández, Daniel, und Stefan Wagner. 2015. «Naming the Pain in Requirements Engineering: A Design for a Global Family of Surveys and First Results from Germany». Information and Software Technology 57 (Januar): 616-43. https://doi.org/10.1016/j. infsof.2014.05.008.

Flick, Uwe. 2002. Qualitative Sozialforschung. Eine Einführung. 6., überarb. und erw. Aufl. Reinbek bei Hamburg: Rowohlt.

Flick, Uwe, Ernst von Kardorff, und Ines Steinke, Hrsg. 2000. Qualitative Forschung. Ein Handbuch. Reinbek: Rowohlt.

Frohlich, David M., und Risto Sarvas. 2011. «HCl and Innovation». In Proceedings of the 2011 Annual Conference Extended Abstracts on Human Factors in Computing Systems - CHI EA '11, 713. Vancouver, BC, Canada: ACM Press. https://doi.org/10.1145/1979742.1979670.

Gervasi, V., R. Gacitua, M. Rouncefield, P. Sawyer, L. Kof, L. Ma, P. Piwek, u. a. 2013. «Unpacking Tacit Knowledge for Requirements Engineering». In Managing Requirements Knowledge, hrsg. v. Walid Maalej und Anil Kumar Thurimella, 23-47. Berlin, Heidelberg: Springer. https://doi.org/10.1007/978-3-642-34419-0_2. 
Hickey, Ann M., und Alan M. Davis. 2004. «A Unified Model of Requirements Elicitation». Journal of Management Information Systems 20 (4): 65-84. https://doi.org/10.1080/07421222.2004 .11045786 .

Holtzblatt, Karen, Jessamyn Burns Wendell, und Shelley Wood. 2004. Rapid Contextual Design. A How-to Guide to Key Techniques for User-Centered Design. San Francisco: Morgan Kaufman.

IDEO LLC. 2013. Design Thinking for Educators. https://designthinkingforeducators.com/toolkit/.

Kamin, Anna-Maria. 2017. «Lernen mit digitalen Medien - Rekonstruktion empirisch emergierter und abduktiv generierter berufsbiographischer Lernmuster». In Projekt - Theorie - Methode. Spektrum medienpädagogischer Forschung, hrsg. v. Thomas Knaus, 317-346, München: koepad.

Kelley, Tom. 2001. The Art of Innovation. Lessons in Creativity from IDEO, America's Leading Design Firm. New York: Doubleday.

Keuneke, Susanne. 2005. «Qualitatives Interview». In Qualitative Medienforschung. Ein Handbuch, hrsg. v. Lothar Mikos und Claudia Wegener. 254-267, Konstanz: UVK.

Knaus, Thomas, Hrsg. 2017. Projekt - Theorie - Methode. Spektrum medienpädagogischer Forschung. München: koepad.

Maiden, Neil, Sara Jones, Kristine Karlsen, Roger Neill, Konstantinos Zachos, und Alastair Milne. 2010. «Requirements Engineering as Creative Problem Solving: A Research Agenda for Idea Finding». In 2010 18th IEEE International Requirements Engineering Conference, 57-66. Sydney, Australia: IEEE. https://doi.org/10.1109/RE.2010.16.

Mey, Günter, und Katja Mruck, Hrsg. 2011. Grounded Theory Reader. Wiesbaden: VS Verlag für Sozialwissenschaften. https://doi.org/10.1007/978-3-531-93318-4.

Meyer, Bertrand. 2014. Agile! The Good, the Hype and the Ugly. Cham: Springer International Publishing. https://doi.org/10.1007/978-3-319-05155-0.

Müller-Benedict, Volker. 2007. Grundkurs Statistik in den Sozialwissenschaften. Eine leicht verständliche, anwendungsorientierte Einführung in das sozialwissenschaftlich notwendige statistische Wissen. 4., Auflage. Wiesbaden: VS Verlag für Sozialwissenschaft/ GWV Fachverlage GmbH. https://doi.org/10.1007/978-3-531-90757-4.

Neuweg, Georg Hans. 1999. Könnerschaft und implizites Wissen, Zur lehr-lerntheoretischen Bedeutung der Erkenntnis- und Wissenstheorie Michael Polanyis. Münster: Waxmann.

Pohl, Klaus. 2007. Requirements Engineering. Heidelberg: dpunkt.

Polanyi, Michael. 1985. Implizites Wissen. Frankfurt: Suhrkamp.

Przyborski, Aglaja, und Monika Wohlrab-Sahr. 2009. Qualitative Sozialforschung. Ein Arbeitsbuch. München: Oldenbourg.

Reinmann, Gabi. 2005. «Innovation ohne Forschung? Ein Plädoyer für den Design-Based Research-Ansatz in der Lehr-Lernforschung». Unterrichtswissenschaft 33 (1): 52-69. http:// nbn-resolving.de/urn:nbn:de:0111-opus-57878. 
Riethmüller, Jürgen. 2012. Der graue Schwan: Prolegomena zum Wissen der Wissensgesellschaft. Paderborn u.a.: Fink.

Strauss, Anselm, und Juliet Corbin. 1996. Grounded Theory. Grundlagen qualitativer Sozialforschung. Weinheim: Beltz.

Sutcliffe, Alistair, und Pete Sawyer. 2013. «Requirements elicitation: Towards the unknown unknowns». In 2013 21st IEEE International Requirements Engineering Conference (RE), 92104. Rio de Janeiro-RJ, Brazil: IEEE. https://doi.org/10.1109/RE.2013.6636709.

Venkatesh, Viswanath, und Hillol Bala. 2008. «Technology Acceptance Model 3 and a Research Agenda on Interventions». Decision Sciences 39 (2): 273-315. https://doi.org/10.1111/ j.1540-5915.2008.00192.x.

Winter, Rainer. 2010. «Handlungsmächtigkeit und technologische Lebensformen: Cultural Studies, digitale Medien und die Demokratisierung der Lebensverhältnisse». In Mensch und Medien. Philosophische und sozialwissenschaftliche Perspektiven, herausgegeben von Manuela Pietraß und Rüdiger Funiok, 139-57. Wiesbaden: VS Verlag für Sozialwissenschaften. https://doi.org/10.1007/978-3-531-92244-7_7.

Wittgenstein, Ludwig. 1984. Über Gewißheit. Frankfurt am Main: Suhrkamp.

Zühlke, Detlef. 2012. Nutzergerechte Entwicklung von Mensch-Maschine-Systemen. UsewareEngineering für technische Systeme. VDI-Buch. Berlin, Heidelberg: Springer. https://doi. org/10.1007/978-3-642-22074-6_1. 